\title{
ERRATUM
}

J. Chil. Chem. Soc., 59, No 4 (2014), page 2701 - 2704

\section{ION PAIR METHOD TO DETERMINE THE CTAB CONTENT IN GOLD NANORODS SAMPLES}

\author{
CAROLINA ADURA ${ }^{1,2}$, FANNY GUZMAN ${ }^{3}$, ALEJANDRO ÁLVAREZ-LUEJE ${ }^{1^{*}}$, MARCELO J. KOGAN1, ${ }^{4^{*}}$ \\ ${ }^{1}$ Departamento de Química Farmacológica y Toxicológica, Facultad de Ciencias Químicas y \\ Farmacéuticas, Universidad de Chile, Santiago, Chile. \\ ${ }^{2}$ Facultad de Química y Biología, Universidad de Santiago de Chile, Santiago, Chile. \\ ${ }^{3}$ Núcleo de Biotecnología Curauma, Pontificia Universidad Católica de Valparaíso, Chile. \\ ${ }^{4}$ Advanced Center for Chronic Diseases (ACCDiS)
}

\section{ACKNOWLEDGMENTS}

This work was supported by Fondecyt 113042, FONDAP 15130011 and MECESUP UCH-0811

Corrected:

\section{ION PAIR METHOD TO DETERMINE THE CTAB CONTENT IN GOLD NANORODS SAMPLES}

CAROLINA ADURA ${ }^{1,2}$, FANNY GUZMAN ${ }^{3}$, ALEJANDRO ÁLVAREZ-LUEJE ${ }^{I^{*}}$, MARCELO J. KOGAN ${ }^{1,4^{*}}$

${ }^{1}$ Departamento de Química Farmacológica y Toxicológica, Facultad de Ciencias Químicas y Farmacéuticas, Universidad de Chile, Santiago, Chile.

${ }^{2}$ Facultad de Química y Biología, Universidad de Santiago de Chile, Santiago, Chile.

${ }^{3}$ Núcleo de Biotecnología Curauma, Pontificia Universidad Católica de Valparaíso, Chile.

${ }^{4}$ Advanced Center for Chronic Diseases (ACCDiS)

\section{ACKNOWLEDGMENTS}

This work was supported by Fondecyt 1130425, FONDAP 15130011 and MECESUP UCH-0811 